\title{
A fast randomized algorithm for overdetermined linear least-squares regression*
}

\author{
Vladimir Rokhlin and Mark Tygert \\ Technical Report YALEU/DCS/TR-1403 \\ April 28, 2008
}

\begin{abstract}
We introduce a randomized algorithm for overdetermined linear least-squares regression. Given an arbitrary full-rank $m \times n$ matrix $A$ with $m \geq n$, any $m \times 1$ vector $b$, and any positive real number $\varepsilon$, the procedure computes an $n \times 1$ vector $x$ which minimizes the spectral norm $\|A x-b\|$ to relative precision $\varepsilon$. The algorithm typically requires $\mathcal{O}\left((\log (n)+\log (1 / \varepsilon)) m n+n^{3}\right)$ floating-point operations. This cost is less than the $\mathcal{O}\left(m n^{2}\right)$ required by the classical schemes based on $Q R$-decompositions or bidiagonalization. We present several numerical examples illustrating the performance of the algorithm.
\end{abstract}

\section{Introduction}

Least-squares fitting has permeated the sciences and engineering following its introduction over two centuries ago (see, for example, [3] for a brief historical review). Linear leastsquares regression is fundamental in the analysis of data, such as that generated from biology, econometrics, engineering, physics, and many other technical disciplines.

Perhaps the most commonly encountered formulation of linear least-squares regression involves a full-rank $m \times n$ matrix $A$ and an $m \times 1$ column vector $b$, with $m \geq n$; the task is to find an $n \times 1$ column vector $x$ such that the spectral norm $\|A x-b\|$ is minimized. Classical algorithms using $Q R$-decompositions or bidiagonalization require

$$
C_{\text {classical }}=\mathcal{O}\left(m n^{2}\right)
$$

floating-point operations in order to compute $x$ (see, for example, [3] or Chapter 5 in [5]). The present paper introduces a randomized algorithm that, given any positive real number $\varepsilon$, computes a vector $x$ minimizing $\|A x-b\|$ to relative precision $\varepsilon$, that is, the algorithm produces a vector $x$ such that

$$
\|A x-b\|-\min _{y \in \mathbb{C}^{n}}\|A y-b\| \leq \varepsilon \min _{y \in \mathbb{C}^{n}}\|A y-b\| .
$$

*Partially supported by ONR Grant \#N00014-07-1-0711, DARPA Grant \#FA9550-07-1-0541, and NGA Grant \#HM1582-06-1-2039. 
This algorithm typically requires

$$
C_{\text {rand }}=\mathcal{O}\left((\log (n)+\log (1 / \varepsilon)) m n+n^{3}\right)
$$

operations. When $n$ is sufficiently large and $m$ is much greater than $n$ (that is, the regression is highly overdetermined), then (3) is less than (1). Furthermore, in the numerical experiments of Section 6 , the algorithm of the present article runs substantially faster than the standard methods based on $Q R$-decompositions.

The method of the present article is an extension of the methods introduced in [7], [8], and [4]. Their algorithms and ours have similar costs; however, for the computation of $x$ minimizing $\|A x-b\|$ to relative precision $\varepsilon$, the earlier algorithms involve costs proportional to $1 / \varepsilon$, whereas the algorithm of the present paper involves a cost proportional to $\log (1 / \varepsilon)$ (see (3) above).

The present article describes algorithms optimized for the case when the entries of $A$ and $b$ are complex valued. Needless to say, real-valued versions of our schemes are similar. This paper has the following structure: Section 2 sets the notation. Section 3 discusses a randomized linear transformation which can be applied rapidly to arbitrary vectors. Section 4 provides the relevant mathematical apparatus. Section 5 describes the algorithm of the present paper. Section 6 illustrates the performance of the algorithm via several numerical examples. Section 7 draws conclusions and proposes directions for future work.

\section{Notation}

In this section, we set notational conventions employed throughout the present paper.

We denote an identity matrix by $\mathbf{1}$. We consider the entries of all matrices in this paper to be complex valued. For any matrix $A$, we define $A^{*}$ to be the adjoint of $A$, and we define the norm $\|A\|$ of $A$ to be the spectral ( $l^{2}$-operator) norm of $A$, that is, $\|A\|$ is the greatest singular value of $A$. We define the condition number of $A$ to be the $l^{2}$ condition number of $A$, that is, the greatest singular value of $A$ divided by the least singular value of $A$. If $A$ has at least as many rows as columns, then the condition number of $A$ is given by the expression

$$
\kappa_{A}=\sqrt{\left\|A^{*} A\right\|\left\|\left(A^{*} A\right)^{-1}\right\|} .
$$

For any positive integers $m$ and $n$ with $m \geq n$, and any $m \times n$ matrix $A$, we will be using the singular value decomposition of $A$ in the form

$$
A_{m \times n}=U_{m \times n} \Sigma_{n \times n} V_{n \times n}^{*},
$$

where $U$ is an $m \times n$ matrix whose columns are orthonormal, $V$ is an $n \times n$ matrix whose columns are orthonormal, and $\Sigma$ is a diagonal $n \times n$ matrix whose entries are all nonnegative. We abbreviate "singular value decomposition" to "SVD" and "independent, identically distributed" to "i.i.d."

For any positive integer $m$, we define the discrete Fourier transform $F^{(m)}$ to be the complex $m \times m$ matrix with the entries

$$
\left(F^{(m)}\right)_{j, k}=\frac{1}{\sqrt{m}} e^{-2 \pi i(j-1)(k-1) / m}
$$


for $j, k=1,2, \ldots, m-1, m$, where $i=\sqrt{-1}$ and $e=\exp (1)$; if the size $m$ is clear from the context, then we omit the superscript in $F^{(m)}$, denoting the discrete Fourier transform by simply $F$.

\section{Preliminaries}

In this section, we discuss a subsampled randomized Fourier transform. [1], [4], [7], and [8] introduced a similar transform for similar purposes.

For any positive integers $l$ and $m$ with $l \leq m$, we define the $l \times m$ SRFT to be the $l \times m$ random matrix

$$
T_{l \times m}=G_{l \times m} H_{m \times m},
$$

where $G$ and $H$ are defined as follows.

In (7), $G$ is the $l \times m$ random matrix given by the formula

$$
G_{l \times m}=S_{l \times m} F_{m \times m} D_{m \times m}
$$

where $S$ is the $l \times m$ matrix whose entries are all zeros, aside from a single 1 in column $s_{j}$ of row $j$ for $j=1,2, \ldots, l-1, l$, where $s_{1}, s_{2}, \ldots, s_{l-1}, s_{l}$ are i.i.d. integer random variables, each distributed uniformly over $\{1,2, \ldots, m-1, m\}$; moreover, $F$ is the $m \times m$ discrete Fourier transform, and $D$ is the diagonal $m \times m$ matrix whose diagonal entries $d_{1}, d_{2}, \ldots$, $d_{m-1}, d_{m}$ are i.i.d. complex random variables, each distributed uniformly over the unit circle. (In our numerical implementations, we drew $s_{1}, s_{2}, \ldots, s_{l-1}, s_{l}$ from $\{1,2, \ldots, m-1, m\}$ without replacement, instead of using i.i.d. draws.)

In (7), $H$ is the $m \times m$ random matrix given by the formula

$$
H_{m \times m}=\Theta_{m \times m} \Pi_{m \times m} Z_{m \times m} \tilde{\Theta}_{m \times m} \tilde{\Pi}_{m \times m} \tilde{Z}_{m \times m}
$$

where $\Pi$ and $\tilde{\Pi}$ are $m \times m$ permutation matrices chosen independently and uniformly at random, and $\underset{Z}{Z}$ and $\tilde{Z}$ are diagonal $m \times m$ matrices whose diagonal entries $\zeta_{1}, \zeta_{2}, \ldots$, $\zeta_{m-1}, \zeta_{m}$ and $\tilde{\zeta}_{1}, \tilde{\zeta}_{2}, \ldots, \tilde{\zeta}_{m-1}, \tilde{\zeta}_{m}$ are i.i.d. complex random variables, each distributed uniformly over the unit circle; furthermore, $\Theta$ and $\tilde{\Theta}$ are the $m \times m$ matrices defined via the formulae

$$
\begin{gathered}
\Theta_{m \times m}=\left(\begin{array}{ccccc}
\cos \left(\theta_{1}\right) & \sin \left(\theta_{1}\right) & 0 & 0 & 0 \\
-\sin \left(\theta_{1}\right) & \cos \left(\theta_{1}\right) & 0 & 0 & 0 \\
0 & 0 & 1 & 0 & 0 \\
0 & 0 & 0 & \ddots & 0 \\
0 & 0 & 0 & 0 & 1
\end{array}\right)\left(\begin{array}{ccccc}
1 & 0 & 0 & 0 & 0 \\
0 & \cos \left(\theta_{2}\right) & \sin \left(\theta_{2}\right) & 0 & 0 \\
0 & -\sin \left(\theta_{2}\right) & \cos \left(\theta_{2}\right) & 0 & 0 \\
0 & 0 & 0 & 1 & 0 \\
0 & 0 & 0 & 0 & \ddots
\end{array}\right) \ldots \\
\ldots\left(\begin{array}{ccccc}
\ddots & 0 & 0 & 0 & 0 \\
0 & 1 & 0 & 0 & 0 \\
0 & 0 & \cos \left(\theta_{m-2}\right) & \sin \left(\theta_{m-2}\right) & 0 \\
0 & 0 & -\sin \left(\theta_{m-2}\right) & \cos \left(\theta_{m-2}\right) & 0 \\
0 & 0 & 0 & 0 & 1
\end{array}\right)\left(\begin{array}{ccccc}
1 & 0 & 0 & 0 & 0 \\
0 & \ddots & 0 & 0 & 0 \\
0 & 0 & 1 & 0 & 0 \\
0 & 0 & 0 & \cos \left(\theta_{m-1}\right) & \sin \left(\theta_{m-1}\right) \\
0 & 0 & 0 & -\sin \left(\theta_{m-1}\right) & \cos \left(\theta_{m-1}\right)
\end{array}\right)
\end{gathered}
$$


and (the same as (10), but with tildes)

$$
\begin{gathered}
\tilde{\Theta}_{m \times m}=\left(\begin{array}{ccccc}
\cos \left(\tilde{\theta}_{1}\right) & \sin \left(\tilde{\theta}_{1}\right) & 0 & 0 & 0 \\
-\sin \left(\tilde{\theta}_{1}\right) & \cos \left(\tilde{\theta}_{1}\right) & 0 & 0 & 0 \\
0 & 0 & 1 & 0 & 0 \\
0 & 0 & 0 & \ddots & 0 \\
0 & 0 & 0 & 0 & 1
\end{array}\right)\left(\begin{array}{ccccc}
1 & 0 & 0 & 0 & 0 \\
0 & \cos \left(\tilde{\theta}_{2}\right) & \sin \left(\tilde{\theta}_{2}\right) & 0 & 0 \\
0 & -\sin \left(\tilde{\theta}_{2}\right) & \cos \left(\tilde{\theta}_{2}\right) & 0 & 0 \\
0 & 0 & 0 & 1 & 0 \\
0 & 0 & 0 & 0 & \ddots
\end{array}\right) \ldots \\
\ldots\left(\begin{array}{ccccc}
\ddots & 0 & 0 & 0 & 0 \\
0 & 1 & 0 & 0 & 0 \\
0 & 0 & \cos \left(\tilde{\theta}_{m-2}\right) & \sin \left(\tilde{\theta}_{m-2}\right) & 0 \\
0 & 0 & -\sin \left(\tilde{\theta}_{m-2}\right) & \cos \left(\tilde{\theta}_{m-2}\right) & 0 \\
0 & 0 & 0 & 0 & 1
\end{array}\right)\left(\begin{array}{ccccc}
1 & 0 & 0 & 0 & 0 \\
0 & \ddots & 0 & 0 & 0 \\
0 & 0 & 1 & 0 & 0 \\
0 & 0 & 0 & \cos \left(\tilde{\theta}_{m-1}\right) & \sin \left(\tilde{\theta}_{m-1}\right) \\
0 & 0 & 0 & -\sin \left(\tilde{\theta}_{m-1}\right) & \cos \left(\tilde{\theta}_{m-1}\right)
\end{array}\right),
\end{gathered}
$$

where $\theta_{1}, \theta_{2}, \ldots, \theta_{m-2}, \theta_{m-1}, \tilde{\theta}_{1}, \tilde{\theta}_{2}, \ldots, \tilde{\theta}_{m-2}, \tilde{\theta}_{m-1}$ are i.i.d. real random variables drawn uniformly from $[0,2 \pi]$. We observe that $\Theta, \tilde{\Theta}, \Pi, \tilde{\Pi}, Z$, and $\tilde{Z}$ are all unitary, and so $H$ is also unitary.

We call the transform $T$ an "SRFT" for lack of a better term.

Remark 3.1 The earlier works [6] and [9] omitted the matrix $H$ in the definition (7) of the SRFT. Numerical experiments indicate that including $H$ improves the performance of the algorithm of the present paper on sparse matrices.

The following lemma is similar to the subspace Johnson-Lindenstrauss lemma (Corollary 11) of [8], and is proven (in a slightly different form) as Lemma 4.4 of [9]. The lemma provides a highly probable upper bound on the condition number of the product of the $l \times m$ matrix $G$ defined in (8) and an independent $m \times n$ matrix $U$ whose columns are orthonormal, assuming that $l$ is less than $m$ and is sufficiently greater than $n^{2}$.

Lemma 3.2 Suppose that $\alpha$ and $\beta$ are real numbers greater than 1 , and $l, m$, and $n$ are positive integers, such that

$$
m>l \geq\left(\frac{\alpha+1}{\alpha-1}\right)^{2} \beta n^{2} .
$$

Suppose further that $G$ is the $l \times m$ random matrix defined in (8). Suppose in addition that $U$ is an $m \times n$ random matrix whose columns are orthonormal, and that $U$ is independent of $G$.

Then, the condition number of $G U$ is at most $\sqrt{\alpha}$ with probability at least $1-\frac{1}{\beta}$.

The following corollary of Lemma 3.2 follows immediately from the fact that the random matrix $H$ defined in (9) is unitary and independent of the random matrix $G$ defined in (8). The corollary provides a highly probable upper bound on the condition number of the $l \times m$ SRFT (defined in (7)) applied to an $m \times n$ matrix $U$ whose columns are orthonormal, assuming that $l$ is less than $m$ and is sufficiently greater than $n^{2}$. 
Corollary 3.3 Suppose that $\alpha$ and $\beta$ are real numbers greater than 1 , and $l, m$, and $n$ are positive integers, such that (12) holds. Suppose further that $T$ is the $l \times m$ SRFT defined in (7). Suppose in addition that $U$ is an $m \times n$ matrix whose columns are orthonormal.

Then, the condition number of $T U$ is at most $\sqrt{\alpha}$ with probability at least $1-\frac{1}{\beta}$.

The following lemma states that, if $A$ is an $m \times n$ matrix, $b$ is an $m \times 1$ vector, and $T$ is the $l \times m$ SRFT defined in (7), then, with high probability, an $n \times 1$ vector $z$ minimizing $\|T A z-T b\|$ also minimizes $\|A z-b\|$ to within a reasonably small factor. Whereas solving $A z \approx b$ in the least-squares sense involves $m$ simultaneous linear equations, solving $T A z \approx$ $T b$ involves just $l$ simultaneous equations. This lemma is modeled after similar results in [4], [7], and [8], and is proven (in a slightly different form) as Lemma 4.8 of [9].

Lemma 3.4 Suppose that $\alpha$ and $\beta$ are real numbers greater than 1 , and $l, m$, and $n$ are positive integers, such that

$$
m>l \geq\left(\frac{\alpha+1}{\alpha-1}\right)^{2} \beta(n+1)^{2} .
$$

Suppose further that $T$ is the $l \times m$ SRFT defined in (7). Suppose in addition that $A$ is an $m \times n$ matrix, and $b$ is an $m \times 1$ vector. Suppose finally that $z$ is an $n \times 1$ vector minimizing the quantity

$$
\|T A z-T b\| .
$$

Then,

$$
\|A z-b\| \leq \sqrt{\alpha} \min _{y \in \mathbb{C}^{n}}\|A y-b\|
$$

\section{Mathematical apparatus}

In this section, we prove a theorem which (in conjunction with Corollary 3.3) guarantees that the algorithm of the present paper is fast.

In the proof of Theorem 4.2 below, we will need the following technical lemma.

Lemma 4.1 Suppose that $l, m$, and $n$ are positive integers such that $m \geq l \geq n$. Suppose further that $A$ is an $m \times n$ matrix, and that the $S V D$ of $A$ is

$$
A_{m \times n}=U_{m \times n} \Sigma_{n \times n} V_{n \times n}^{*},
$$

where $U$ is an $m \times n$ matrix whose columns are orthonormal, $V$ is an $n \times n$ matrix whose columns are orthonormal, and $\Sigma$ is a diagonal $n \times n$ matrix whose entries are all nonnegative. Suppose in addition that $T$ is an $l \times m$ matrix, and that the $S V D$ of the $l \times n$ matrix TU is

$$
T_{l \times m} U_{m \times n}=\tilde{U}_{l \times n} \tilde{\Sigma}_{n \times n} \tilde{V}_{n \times n}^{*} .
$$

Then, there exist an $n \times n$ matrix $P$, and an $l \times n$ matrix $Q$ whose columns are orthonormal, such that

$$
T_{l \times m} A_{m \times n}=Q_{l \times n} P_{n \times n}
$$


Furthermore, if $P$ is any $n \times n$ matrix, and $Q$ is any $l \times n$ matrix whose columns are orthonormal, such that $P$ and $Q$ satisfy (18), then

$$
P_{n \times n}=\left(Q_{l \times n}\right)^{*} \tilde{U}_{l \times n} \tilde{\Sigma}_{n \times n} \tilde{V}_{n \times n}^{*} \Sigma_{n \times n} V_{n \times n}^{*} ;
$$

if, in addition, the matrices $A$ and $T U$ both have full rank (rank $n$ ), then there exists a unitary $n \times n$ matrix $W$ such that

$$
\tilde{U}_{l \times n}=Q_{l \times n} W_{n \times n} .
$$

Proof. An example of matrices $P$ and $Q$ satisfying (18) such that the columns of $Q$ are orthonormal is $P=\tilde{\Sigma} \tilde{V}^{*} \Sigma V^{*}$ and $Q=\tilde{U}$.

We now assume that $P$ is any $n \times n$ matrix, and $Q$ is any $l \times n$ matrix whose columns are orthonormal, such that $P$ and $Q$ satisfy (18). Combining (18), (16), and (17) yields

$$
Q_{l \times n} P_{n \times n}=\tilde{U}_{l \times n} \tilde{\Sigma}_{n \times n} \tilde{V}_{n \times n}^{*} \Sigma_{n \times n} V_{n \times n}^{*} .
$$

Combining (21) and the fact that the columns of $Q$ are orthonormal (so that $Q^{*} Q=\mathbf{1}$ ) yields (19).

For the remainder of the proof, we assume that the matrices $A$ and $T U$ both have full rank. To establish (20), we demonstrate that the column spans of $Q$ and $\tilde{U}$ are the same. It then follows from the fact that the columns of $Q$ are an orthonormal basis for this column span, as are the columns of $\tilde{U}$, that there exists a unitary $n \times n$ matrix $W$ satisfying (20). We now complete the proof by showing that

$$
\text { column span of } Q_{l \times n}=\text { column span of } \tilde{U}_{l \times n} .
$$

Obviously, it follows from (21) that

$$
\text { column span of } Q_{l \times n} P_{n \times n}=\text { column span of } \tilde{U}_{l \times n} \tilde{\Sigma}_{n \times n} \tilde{V}_{n \times n}^{*} \Sigma_{n \times n} V_{n \times n}^{*} \text {; }
$$

we will simplify both sides of (23), in order to obtain (22).

It follows from the fact that $A$ and $T U$ both have full rank that the matrices $\Sigma$ and $\tilde{\Sigma}$ in the SVDs (16) and (17) are nonsingular, and so (as the unitary matrices $V$ and $\tilde{V}$ are also nonsingular)

$$
\text { column span of } \tilde{U}_{l \times n} \tilde{\Sigma}_{n \times n} \tilde{V}_{n \times n}^{*} \Sigma_{n \times n} V_{n \times n}^{*}=\text { column span of } \tilde{U}_{l \times n} .
$$

Combining (23), (24), and the fact that the column span of $\tilde{U}$ is $n$-dimensional (after all, the $n$ columns of $\tilde{U}$ are orthonormal) yields that the column span of $Q P$ is $n$-dimensional. Combining this fact, the fact that the column span of $Q P$ is a subspace of the column span of $Q$, and the fact that the column span of $Q$ is $n$-dimensional (after all, the $n$ columns of $Q$ are orthonormal) yields

$$
\text { column span of } Q_{l \times n} P_{n \times n}=\text { column span of } Q_{l \times n} \text {. }
$$

Combining (23), (24), and (25) yields (22).

The following theorem states that, given an $m \times n$ matrix $A$, the condition number of a certain preconditioned version of $A$ corresponding to an $l \times m$ matrix $T$ is equal to the condition number of $T U$, where $U$ is an $m \times n$ matrix of orthonormal left singular vectors of $A$. 
Theorem 4.2 Suppose that $l, m$, and $n$ are positive integers such that $m \geq l \geq n$. Suppose further that $A$ is a full-rank $m \times n$ matrix, and that the $S V D$ of $A$ is

$$
A_{m \times n}=U_{m \times n} \Sigma_{n \times n} V_{n \times n}^{*} .
$$

Suppose in addition that $T$ is an $l \times m$ matrix such that the $l \times n$ matrix $T U$ has full rank.

Then, there exist an $n \times n$ matrix $P$, and an $l \times n$ matrix $Q$ whose columns are orthonormal, such that

$$
T_{l \times m} A_{m \times n}=Q_{l \times n} P_{n \times n} .
$$

Furthermore, if $P$ is any $n \times n$ matrix, and $Q$ is any $l \times n$ matrix whose columns are orthonormal, such that $P$ and $Q$ satisfy (27), then the condition numbers of $A P^{-1}$ and $T U$ are equal.

Proof. Lemma 4.1 guarantees the existence of matrices $P$ and $Q$ satisfying (27) such that the columns of $Q$ are orthonormal.

For the remainder of the proof, we assume that $P$ is an $n \times n$ matrix, and $Q$ is an $l \times n$ matrix whose columns are orthonormal, such that $P$ and $Q$ satisfy (27). Combining (19), (20), and the fact that the columns of $Q$ are orthonormal (so that $Q^{*} Q=1$ ) yields

$$
P_{n \times n}=W_{n \times n} \tilde{\Sigma}_{n \times n} \tilde{V}_{n \times n}^{*} \Sigma_{n \times n} V_{n \times n}^{*}
$$

where $W$ is the matrix from (20), and $\tilde{\Sigma}$ and $\tilde{V}$ are the matrices from the SVD (17). Combining (26), (28), and the fact that $V, \tilde{V}$, and $W$ are unitary yields

$$
A_{m \times n} P_{n \times n}^{-1}=U_{m \times n} \tilde{V}_{n \times n} \tilde{\Sigma}_{n \times n}^{-1} W_{n \times n}^{*} .
$$

Combining (29), the fact that $\tilde{V}$ and $W$ are unitary, the fact that the columns of $U$ are orthonormal (so that $U^{*} U=\mathbf{1}$ ), and the fact that $\tilde{\Sigma}$ is diagonal yields

$$
\left\|\left(A_{m \times n} P_{n \times n}^{-1}\right)^{*}\left(A_{m \times n} P_{n \times n}^{-1}\right)\right\|=\left\|\tilde{\Sigma}_{n \times n}^{-1}\right\|^{2}
$$

and

$$
\left\|\left(\left(A_{m \times n} P_{n \times n}^{-1}\right)^{*}\left(A_{m \times n} P_{n \times n}^{-1}\right)\right)^{-1}\right\|=\left\|\tilde{\Sigma}_{n \times n}\right\|^{2} .
$$

Combining (4), (30), (31), and the SVD (17) yields the present theorem.

\section{The algorithm}

In this section, we describe the algorithm of the present paper, giving details about its implementation and computational costs. 


\subsection{Description of the algorithm}

Suppose that $m$ and $n$ are positive integers with $m \geq n, A$ is a full-rank $m \times n$ matrix, and $b$ is an $m \times 1$ column vector. In this subsection, we describe a procedure for the computation of an $n \times 1$ column vector $x$ such that $\|A x-b\|$ is minimized to arbitrarily high precision.

Rather than directly calculating the vector $x$ minimizing $\|A x-b\|$, we will first calculate the vector $y$ minimizing $\|C y-b\|$, where $C=A P^{-1}$ and $y=P x$, with an appropriate choice of an $n \times n$ matrix $P$; the matrix $P$ is known as a preconditioning matrix. With an appropriate choice of $P$, the condition number of $C$ is reasonably small, and so an iterative solver such as the conjugate gradient method will require only a few iterations in order to obtain $y$ minimizing $\|C y-b\|$ to high precision. Once we have calculated $y$, we obtain $x$ via the formula $x=P^{-1} y$.

To construct the preconditioning matrix $P$, we compute $Y=T A$, where $T$ is the $l \times m$ SRFT defined in (7), with $m \geq l \geq n$. We then form a pivoted $Q R$-decomposition of $Y$, computing an $l \times n$ matrix $Q$ whose columns are orthonormal, an upper-triangular $n \times n$ matrix $R$, and an $n \times n$ permutation matrix $\Pi$, such that $Y=Q R \Pi$. We use the product $P=R \Pi$ as the preconditioning matrix. Fortuitously, since this matrix $P$ is the product of an upper-triangular matrix and a permutation matrix, we can apply $P^{-1}$ or $\left(P^{-1}\right)^{*}$ to any arbitrary vector rapidly, without calculating the entries of $P^{-1}$ explicitly.

The condition number of $C=A P^{-1}$ is reasonably small with very high probability whenever $l$ is sufficiently greater than $n$, due to Theorem 4.2 and Corollary 3.3; moreover, numerical experiments in Section 6 suggest that the condition number of $C$ is practically always less than 3 or so when $l=4 n$. Therefore, when $l$ is sufficiently greater than $n$, the conjugate gradient method requires only a few iterations in order to compute $y$ minimizing $\|C y-b\|$ to high precision; furthermore, the conjugate gradient method requires only applications of $A, A^{*}, P^{-1}$, and $\left(P^{-1}\right)^{*}$ to vectors, and all of these matrices are readily available for application to vectors. Once we have calculated $y$, we obtain $x$ minimizing $\|A x-b\|$ via the formula $x=P^{-1} y$.

There is a natural choice for the starting vector of the conjugate gradient iterations. Combining the fact that $Y=T A$ with (15) yields that, with high probability, the $n \times 1$ vector $z$ minimizing $\|Y z-T b\|$ also minimizes $\|A z-b\|$ to within a factor of 3 , provided $l$ is sufficiently greater than $n$ (in practice, $l=4 n$ is sufficient). Thus, $z$ is a good choice for the starting vector. Moreover, combining the facts that $Y=Q P$ and that the columns of $Q$ are orthonormal yields that $z=P^{-1} Q^{*} T b$, providing a convenient means of computing $z$.

In summary, if $\varepsilon$ is any specified positive real number, we can compute an $n \times 1$ column vector $x$ which minimizes $\|A x-b\|$ to relative precision $\varepsilon$ via the following five steps:

1. Compute $Y=T A$, where $T$ is the $l \times m$ SRFT defined in (7), with $m \geq l \geq n$. (See, for example, Subsection 3.3 of [9] for details on applying the SRFT rapidly.)

2. Form a pivoted $Q R$-decomposition of $Y$ from Step 1, computing an $l \times n$ matrix $Q$ whose columns are orthonormal, an upper-triangular $n \times n$ matrix $R$, and an $n \times n$ permutation matrix $\Pi$, such that $Y=Q R \Pi$. (See, for example, Chapter 5 in [5] for details on computing such a pivoted $Q R$-decomposition.)

3. Compute the $n \times 1$ column vector $z=P^{-1}\left(Q^{*}(T b)\right)$, where $T$ is the $l \times m$ SRFT defined in (7), $Q$ is the $l \times n$ matrix from Step 2 whose columns are orthonormal, and 
$P=R \Pi ; R$ and $\Pi$ are the upper-triangular and permutation matrices from Step 2. (See, for example, Subsection 3.3 of [9] for details on applying the SRFT rapidly.)

4. Compute an $n \times 1$ column vector $y$ which minimizes $\left\|A P^{-1} y-b\right\|$ to relative precision $\varepsilon$, via the preconditioned conjugate gradient iterations, where $P=R \Pi$ is the preconditioning matrix; $R$ and $\Pi$ are the upper-triangular and permutation matrices from Step 2. Use $z$ from Step 3 as the starting vector. (See, for example, Algorithm 7.4.3 in [3] for details on the preconditioned conjugate gradient iterations for linear leastsquares problems.)

5. Compute $x=P^{-1} y$, where $y$ is the vector from Step 4, and again $P=R \Pi ; R$ and $\Pi$ are the upper-triangular and permutation matrices from Step 2.

\subsection{Cost}

In this subsection, we estimate the number of floating-point operations required by each step of the algorithm of the preceding subsection.

We denote by $\kappa$ the condition number of the preconditioned matrix $A P^{-1}$. The five steps of the algorithm incur the following costs:

1. Applying $T$ to every column of $A \operatorname{costs} \mathcal{O}(m n \log (l))$.

2. Computing the pivoted $Q R$-decomposition of $Y \operatorname{costs} \mathcal{O}\left(n^{2} l\right)$.

3. Applying $T$ to $b \operatorname{costs} \mathcal{O}(m \log (l))$. Applying $Q^{*}$ to $T b \operatorname{costs} \mathcal{O}(n l)$. Applying $P^{-1}=$ $\Pi^{-1} R^{-1}$ to $Q^{*} T b \operatorname{costs} \mathcal{O}\left(n^{2}\right)$.

4. When $l \geq 4 n^{2}$, (15) guarantees with high probability that the vector $z$ has a residual $\|A z-b\|$ that is no greater than 3 times the minimum possible. When started with such a vector, the preconditioned conjugate gradient algorithm requires $\mathcal{O}(\kappa \log (1 / \varepsilon))$ iterations in order to improve the relative precision of the residual to $\varepsilon$ (see, for example, formula 7.4.7 in [3]). Applying $A$ and $A^{*}$ a total of $\mathcal{O}(\kappa \log (1 / \varepsilon))$ times costs $\mathcal{O}(m n \kappa \log (1 / \varepsilon))$. Applying $P^{-1}$ and $\left(P^{-1}\right)^{*}$ a total of $\mathcal{O}(\kappa \log (1 / \varepsilon))$ times costs $\mathcal{O}\left(n^{2} \kappa \log (1 / \varepsilon)\right)$. These costs dominate the costs of the remaining computations in the preconditioned conjugate gradient iterations.

5. Applying $P^{-1}=\Pi^{-1} R^{-1}$ to $y \operatorname{costs} \mathcal{O}\left(n^{2}\right)$.

Summing up the costs in the five steps above, we see that the cost of the entire algorithm is

$$
C_{\text {theoretical }}=\mathcal{O}\left((\log (l)+\kappa \log (1 / \varepsilon)) m n+n^{2} l\right) .
$$

The condition number $\kappa$ of the preconditioned matrix $A P^{-1}$ can be made arbitrarily close to 1 , by choosing $l$ sufficiently large. According to Theorem 4.2 and Corollary 3.3, choosing $l \geq 4 n^{2}$ guarantees that $\kappa$ is at most 3 , with high probability. 
Remark 5.1 Currently, our estimates require that $l$ be at least $4 n^{2}$ in order to ensure with high probability that $\kappa$ is at most 3 and that the residual $\|A z-b\|$ is no greater than 3 times the minimum possible. However, our numerical experiments indicate that it is not necessary for $l$ to be as large as $4 n^{2}$ (though it is sufficient). Indeed, in all of our tests, choosing $l=4 n$ produced a condition number $\kappa$ less than 3 and a residual $\|A z-b\|$ no greater than 3 times the minimum possible residual. With $l=4 n$ and $\kappa \leq 3$, the cost (32) becomes

$$
C_{\text {typical }}=\mathcal{O}\left((\log (n)+\log (1 / \varepsilon)) m n+n^{3}\right) .
$$

\section{Numerical results}

In this section, we describe the results of several numerical tests of the algorithm of the present paper.

We use the algorithm to compute an $n \times 1$ vector $x$ minimizing $\|A x-b\|$ to high precision, where $b$ is an $m \times 1$ vector, and $A$ is the $m \times n$ matrix defined via the formula

$$
A_{m \times n}=U_{m \times n} \Sigma_{n \times n} V_{n \times n}^{*} ;
$$

in all experiments reported below, $U$ is obtained by applying the Gram-Schmidt process to the columns of an $m \times n$ matrix whose entries are i.i.d. centered complex Gaussian random variables, $V$ is obtained by applying the Gram-Schmidt process to the columns of an $n \times n$ matrix whose entries are i.i.d. centered complex Gaussian random variables, and $\Sigma$ is a diagonal $n \times n$ matrix, with the diagonal entries

$$
\Sigma_{k, k}=10^{-6(k-1) /(n-1)}
$$

for $k=1,2, \ldots, n-1, n$. Clearly, the condition number $\kappa_{A}$ of $A$ is

$$
\kappa_{A}=\Sigma_{1,1} / \Sigma_{n, n}=10^{6} .
$$

The $m \times 1$ unit vector $b$ is defined via the formula

$$
b=10^{-3} w+A x,
$$

where $w$ is a random $m \times 1$ unit vector orthogonal to the column span of $A$, and $A x$ is a vector from the column span of $A$ such that $\|b\|=1$.

We implemented the algorithm in Fortran 77 in double-precision arithmetic, and used the Lahey/Fujitsu Express v6.2 compiler. We used one core of a $1.86 \mathrm{GHz}$ Intel Centrino Core Duo microprocessor with 1 GB of RAM. For the direct computations, we used the classical algorithm for pivoted $Q R$-decompositions based on plane (Householder) reflections (see, for example, Chapter 5 in [5]).

Table 1 displays timing results with $m=32768$ for various values of $n$; Table 2 displays the corresponding errors. Table 3 displays timing results with $n=256$ for various values of $m$; Table 4 displays the corresponding errors.

The headings of the tables are as follows:

- $m$ is the number of rows in the matrix $A$, as well as the length of the vector $b$, in $\|A x-b\|$. 
- $n$ is the number of columns in the matrix $A$, as well as the length of the vector $x$, in $\|A x-b\|$.

- $l$ is the number of rows in the matrix $T$ used in Steps 1 and 3 of the procedure of Subsection 5.1.

- $t_{\text {direct }}$ is the time in seconds required by the classical algorithm.

- $t_{\mathrm{rand}}$ is the time in seconds required by the algorithm of the present paper.

- $t_{\text {direct }} / t_{\text {rand }}$ is the factor by which the algorithm of the present paper is faster than the classical algorithm.

- $\kappa$ is the condition number of $A P^{-1}$, the preconditioned version of the matrix $A$.

- $i$ is the number of iterations required by the preconditioned conjugate gradient method to yield the requested precision $\varepsilon_{\text {rel }}$ of $.5 \mathrm{E}-14$ or better in Table 2 , and $.5 \mathrm{E}-10$ or better in Table 4.

- $\varepsilon_{\text {rel }}$ is defined via the formula

$$
\varepsilon_{\mathrm{rel}}=\frac{\delta-\delta_{\min }}{\kappa_{A} \cdot \delta_{\min }}
$$

where $\kappa_{A}$ is the condition number of $A$ given in (36), $\delta=\|A x-b\|$ ( $x$ is the solution vector produced by the randomized algorithm), and

$$
\delta_{\min }=\min _{y \in \mathbb{C}^{n}}\|A y-b\|=10^{-3} .
$$

Remark 6.1 Standard perturbation theory shows that $\varepsilon_{\text {rel }}$ is the appropriately normalized measure of the precision produced by the algorithm; see, for example, formula 1.4.27 in [3].

The values for $\varepsilon_{\text {rel }}$ and $i$ reported in the tables are the worst (maximum) values encountered during 10 independent randomized trials of the algorithm, as applied to the same matrix $A$. The values for $t_{\text {rand }}$ reported in the tables are the average values over 10 independent randomized trials. None of the quantities reported in the tables varied significantly over repeated randomized trials.

The following observations can be made from the examples reported here, and from our more extensive experiments:

1. When $m=32768$ and $n=512$, the randomized algorithm runs over 5 times faster than the classical algorithm based on plane (Householder) reflections, even at full double precision.

2. As observed in Remark 5.1, our choice $l=4 n$ seems to ensure that the condition number $\kappa$ of the preconditioned matrix is at most 3. More generally, $\kappa$ seems to be less than a function of the ratio $l / n$.

3. The algorithm of the present paper attains high precision at reasonably low cost. 


\section{Conclusions and generalizations}

This article provides a fast algorithm for overdetermined linear least-squares regression. If the matrices $A$ and $A^{*}$ from the regression involving $\|A x-b\|$ can be applied sufficiently rapidly to arbitrary vectors, then the algorithm of the present paper can be accelerated further. Moreover, the methods developed here for overdetermined regression extend to underdetermined regression.

The theoretical bounds in Lemma 3.2, Corollary 3.3, and Lemma 3.4 should be considered preliminary. Our numerical experiments indicate that the algorithm of the present article performs better than our estimates guarantee. Furthermore, there is nothing magical about the subsampled randomized Fourier transform defined in (7). In our experience, several other similar transforms appear to work at least as well, and we are investigating these alternatives (see, for example, [2]).

\section{Acknowledgements}

We would like to thank Franco Woolfe for helpful discussions. 
Table 1:

\begin{tabular}{c|c|c|c|c|c}
$m$ & $n$ & $l$ & $t_{\text {direct }}$ & $t_{\text {rand }}$ & $t_{\text {direct }} / t_{\text {rand }}$ \\
\hline \hline 32768 & 64 & 256 & $.14 \mathrm{E}+01$ & $.13 \mathrm{E}+01$ & 1.1 \\
\hline 32768 & 128 & 512 & $.55 \mathrm{E}+01$ & $.27 \mathrm{E}+01$ & 2.0 \\
\hline 32768 & 256 & 1024 & $.22 \mathrm{E}+02$ & $.59 \mathrm{E}+01$ & 3.7 \\
\hline 32768 & 512 & 2048 & $.89 \mathrm{E}+02$ & $.15 \mathrm{E}+02$ & 5.7 \\
\hline
\end{tabular}

Table 2:

\begin{tabular}{c|c|c|c|c|c}
$m$ & $n$ & $l$ & $\kappa$ & $i$ & $\varepsilon_{\text {rel }}$ \\
\hline \hline 32768 & 64 & 256 & 2.7 & 14 & $.120 \mathrm{E}-15$ \\
\hline 32768 & 128 & 512 & 2.9 & 14 & $.132 \mathrm{E}-15$ \\
\hline 32768 & 256 & 1024 & 2.9 & 14 & $.429 \mathrm{E}-15$ \\
\hline 32768 & 512 & 2048 & 2.9 & 13 & $.115 \mathrm{E}-14$ \\
\hline
\end{tabular}

Table 3:

\begin{tabular}{c|c|c|c|c|c}
$m$ & $n$ & $l$ & $t_{\text {direct }}$ & $t_{\text {rand }}$ & $t_{\text {direct }} / t_{\text {rand }}$ \\
\hline \hline 2048 & 256 & 1024 & $.12 \mathrm{E}+01$ & $.71 \mathrm{E}+00$ & 1.6 \\
\hline 4096 & 256 & 1024 & $.25 \mathrm{E}+01$ & $.94 \mathrm{E}+00$ & 2.6 \\
\hline 8192 & 256 & 1024 & $.51 \mathrm{E}+01$ & $.14 \mathrm{E}+01$ & 3.5 \\
\hline 16384 & 256 & 1024 & $.10 \mathrm{E}+02$ & $.26 \mathrm{E}+01$ & 4.1 \\
\hline 32768 & 256 & 1024 & $.22 \mathrm{E}+02$ & $.50 \mathrm{E}+01$ & 4.4 \\
\hline 65536 & 256 & 1024 & $.49 \mathrm{E}+02$ & $.11 \mathrm{E}+02$ & 4.4 \\
\hline
\end{tabular}

Table 4:

\begin{tabular}{c|c|c|c|c|c}
$m$ & $n$ & $l$ & $\kappa$ & $i$ & $\varepsilon_{\text {rel }}$ \\
\hline \hline 2048 & 256 & 1024 & 2.2 & 4 & $.326 \mathrm{E}-10$ \\
\hline 4096 & 256 & 1024 & 2.6 & 5 & $.364 \mathrm{E}-10$ \\
\hline 8192 & 256 & 1024 & 2.7 & 6 & $.160 \mathrm{E}-10$ \\
\hline 16384 & 256 & 1024 & 2.8 & 7 & $.599 \mathrm{E}-11$ \\
\hline 32768 & 256 & 1024 & 2.9 & 8 & $.502 \mathrm{E}-11$ \\
\hline 65536 & 256 & 1024 & 2.9 & 8 & $.177 \mathrm{E}-11$ \\
\hline
\end{tabular}




\section{References}

[1] N. Ailon And B. Chazelle, Approximate nearest neighbors and the fast Johnson-Lindenstrauss transform, SIAM J. Comput., (2007). To appear.

[2] N. Ailon And E. Liberty, Fast dimension reduction using Rademacher series on dual BCH codes, Tech. Rep. 1385, Yale University Department of Computer Science, July 2007.

[3] A. BJÖRCK, Numerical Methods for Least Squares Problems, SIAM, 1996.

[4] P. Drineas, M. W. Mahoney, S. Muthukrishnan, and T. Sarlós, Faster least squares approximation, Tech. Rep. 0710.1435, arXiv, October 2007. Available at http://arxiv.org/.

[5] G. H. Golub and C. F. Van Loan, Matrix Computations, Johns Hopkins University Press, Baltimore, Maryland, third ed., 1996.

[6] E. Liberty, F. Woolfe, P.-G. Martinsson, V. Rokhlin, and M. Tygert, Randomized algorithms for the low-rank approximation of matrices, Proc. Natl. Acad. Sci. USA, 104 (2007), pp. 20167-20172.

[7] T. SARLós, Improved approximation algorithms for large matrices via random projections, in Proceedings of FOCS 2006, the 47th Annual IEEE Symposium on Foundations of Computer Science, October 2006.

[8] _ Improved approximation algorithms for large matrices via random projections, revised, extended long form. Manuscript in preparation for publication, currently available at http://www.ilab.sztaki.hu/ stamas/publications/rp-long.pdf, 2006.

[9] F. Woolfe, E. Liberty, V. Rokhlin, And M. Tygert, A fast randomized algorithm for the approximation of matrices, Appl. Comput. Harmon. Anal., (2007). To appear. 